\title{
ENTRAMADOS HUMANO-NATURALES COMO RUTA POSIBLE HACIA LA TRANSDISCIPLINARIEDAD EN EL CAMPO DE LA ECOLOGÍA HUMANA Y SABERES AMBIENTALES DE LA UNIVERSIDAD DE CALDAS
}

\author{
Jorge Sánchez-Maldonado \\ Recibido el 26 de septiembre de 2015, aprobado el 2 de mayo de 2016 y actualizado el \\ 22 de diciembre de 2016
}

DOI: 10.17151/luaz.2017.44.16

\section{Resumen}

En el presente escrito me aproximo a la transdisciplinariedad y la necesidad que tenemos de abordarla desde la maestría en Ecología Humana y Saberes Ambientales de la Universidad de Caldas. Para ello, me remito a la exposición de dos referentes teóricos críticos de la modernidad que ponen de manifiesto dos cosas: en primer lugar, las tensiones existentes entre ciencias sociales y ciencias naturales (fundamentales en nuestra formación) y la necesidad de superarlas, en segundo lugar y no menos importante, las relaciones de poder y los procesos que han hecho que estas dos vertientes de la producción de conocimientos invisibilicen otros sistemas de prácticas y conocimientos presentes en sujetos no-tan-modernos (Martínez-Dueñas, 2012). Se plantea que observar entramados humano-naturales (Escobar, 2013) y la ontología política de los problemas ecológicos-humanos, sugieren un camino adecuado para avanzar hacia la construcción de agendas transdisciplinarias de investigación en ecología humana y saberes ambientales.

Palabras clave: entramados humano-naturales, inter-disciplinariedad, ontología política, transdisciplinariedad.

\section{HUMAN-NATURAL WEB AS POSSIBLE ROUTE TOWARDS TRANSDISCIPLINARITY IN THE FIELD OF HUMAN ECOLOGY AND ENVIRONMENTAL KNOWLEDGE AT UNIVERSIDAD DE CALDAS}

\begin{abstract}
This paper offers an approach to transdisciplinarity and the need to engage it in the Human Ecology and Environmental Knowledge Master's Program at Universidad de Caldas, Colombia. To do this, two critical theoretical perspectives on the modernity are presented which illustrate, first, the tension existing between Social and Natural Sciences (essential in the academic background), and the need to overcome them, and secondly and equally important, the power relationships and the process that have made these two aspects of knowledge production to make invisible other systems of practice of knowledge present in not-so-modern actors (Martínez-Dueñas, 2012). This study proposes that observing human-natural webs (Escobar, 2013) and a political ontology of human-ecological problems suggest the adequate route to advance towards the construction of transdisciplinary research agendas in human ecology and environmental knowledge.
\end{abstract}

Keywords: human-natural networks, interdisciplinary nature, political ontology, transdisciplinarity. 
Peces, moluscos, manglares, oxígeno, secuestro de carbono, manejo del territorio, biodiversidad. Gente, peces, moluscos, mamíferos, más gente y manglar, canoas, recorridos, remos y palancas (de manglar), territorio, conocimientos ancestrales, economías de otro modo, cultura, luchas ambientales. Ecosistemas, ciencia, biología, ecología, educación, trabajo social, economía, intelectuales militantes, activistas, filosofía, antropología, discursos, políticas, más mamíferos, conflictos, relaciones interespecies, veganismo, saberes ambientales, prácticas científicas, prácticas otras. Una lista de este tipo -aunque podría ser más amplia- describe una parte del ámbito y las problemáticas asociadas a las actividades de investigación y formación de algunos de los integrantes del grupo de estudiantes de Maestría en Ecología Humana y Saberes Ambientales de la Universidad de Caldas, Manizales. Elementos diferentes y de diferentes naturalezas, aparentemente aislados, habitantes y actantes de parcelas hasta ahora asignadas tanto a las ciencias sociales como a las ciencias naturales, mediante la división disciplinaria del trabajo orientado a la producción de conocimientos. Estos actantes ${ }^{2}$ cobran sentido en cada uno de los espacios de actividad de los investigadores en formación que son biólogos, químicos, filósofos, administradores, antropólogos y sociólogos.

La comprensión de las relaciones conflictivas entre esas parcelas del conocimiento, así como la superación de aquello llamado "racionalidad disciplinaria" (Lanz, 2010), constituyen el punto de partida de la presente reflexión. Ese trabajo no es algo novedoso, ya se han adelantado muchos esfuerzos para caminar hacia aquello que llamaremos aquí, de la mano de otros autores, "transdisciplinariedad". En este sentido, intento avanzar en la búsqueda de lineamientos de trabajo orientados a la transdisciplinariedad como principio metodológico, ético, político y epistemológico de investigación-acción. Lanz (2010) argumenta que no podemos confundir la crítica radical epistemológica a la racionalidad disciplinaria de la modernidad, con la "negación mecánica" de los conocimientos producidos por esas disciplinas, porque implicaría un desconocimiento a los esfuerzos que la humanidad ha emprendido al tratar de comprender el mundo.

$\mathrm{Y}$ es en ese comprender el mundo que la transdisciplinariedad adquiere la mayor importancia. Implica pasar de una comprensión simplista/especializada, a una compleja. Lanz (2010) considera fundamental dar varios pasos para avanzar en esa dirección: primero, conocer la crisis de la modernidad y del discurso cientificista tradicional, lo cual implica reconocer que existen al menos dos vertientes críticas epistemológicas sobre la modernidad y el estatuto cientificista: por un lado, las críticas que se hacen a las disciplinas científicas en particular, y por otro, las críticas hechas en términos generales a las bases del conocimiento científico. Luego de comprender estas críticas -recomienda el autor-, es necesario establecer una cierta distancia de ello, procurando avanzar hacia la línea que supera las fronteras entre las disciplinas. Podría adicionarse a estos movimientos, el de iniciar la apertura de condiciones de posibilidad para diálogos inter-epistémicos con grupos humanos a quienes la modernidad occidental negó la posibilidad y validez de sus conocimientos y experiencias, es decir, lo que en términos de Boaventura de Sousa Santos (2010) consistiría en promover el diálogo de saberes y la lucha contra el desperdicio de la experiencia de otros pueblos dado que -parafraseándolo- "la comprensión del mundo es mucho más que la comprensión occidental del mundo” (2010). 
En este sentido, este escrito debería asimilarse a un camino que se inicia con la mejor intensión de divagar, andar y comprender el terreno de la transdisciplinariedad. En un primer momento, vale la pena tener en cuenta la complicidad de las ciencias sociales con proyectos imperiales que, como veremos, afectaron no solamente a los grupos humanos colonizados sino también, en el marco de esa relación, también a seres nohumanos y a lo que conocemos como "naturaleza". Todo esto se dio en el proceso de formación de una economía-mundo en el que la integración de vastas zonas de la periferia a la economía de los centros metropolitanos implicó la puesta en marcha de formas de explotación y trabajo coercitivo sobre poblaciones humanas africanas e indígenas -para el caso de Las Américas- que varios autores ilustran bien (Wallerstein, 1998; Quijano, 2000; Wallerstein y Quijano, 1992; Mintz, 1996) ${ }^{3}$.

A este respecto, Quijano (2000) acuña la noción de colonialidad del poder, para referirse al patrón de poder capitalista que se hace mundial y hegemónico a partir de América en 1492, al que le siguen, en el mismo periodo de tiempo, el establecimiento de la modernidad y la colonialidad como elementos constitutivos de su funcionamiento. Según el autor, este patrón de poder mundial capitalista se basa en una clasificación racial/étnica que genera la conformación de las "nuevas identidades societales de la colonialidad" (indios, negros, aceitunados, amarillos, blancos, mestizos) y las "identidades geoculturales del colonialismo" (América, África, Lejano Oriente, Cercano Oriente) (2000). No es por casualidad -nos dice el autor-, que en los centros hegemónicos de ese nuevo patrón de poder mundial (es decir, países como Holanda y Francia con Descartes e Inglaterra con Locke y Newton), emerja una determinada manera de producir conocimientos que daba cuenta de necesidades tales como medición, cuantificación, entre otras que se venían presentando para la dominación y explotación de los nuevos territorios conquistados. Este tipo de conocimiento fue asumido como el "conocimiento racional" (Ibíd.), al que sujetos tales como los indios y negros entre otros, no tenían posibilidad de acceder y mucho menos producir, aunque se presentaran en este contexto respuestas al horror de la modernidad que autores como Nelson Maldonado Torres (2008) ha denominado "actitud decolonial", la cual implicaría, en términos de conocimientos, formas de resistencia a esos cánones impuestos desde la modernidad occidental. Mignolo (2008) resalta aquí ejemplos como Ottobah Cugoano para el caso de la lucha de descendientes africanos y a Felipe Guamán Poma de Ayala, para el caso indígena (2008). Sin embargo, éstos no son los únicos ejemplos de la actitud decolonial, así que parte del trabajo está en entablar el diálogo con esos Otros que producen otros conocimientos críticos a partir de su experiencia.

A Quijano se adhieren distintos investigadores que extienden la noción de colonialidad del poder a otros ámbitos de investigación, tales como la producción de conocimientos por un lado (Lander, 2002; Palermo, 2010), y las relaciones de poder en las que se ven inmersos sujetos racializados en distintas partes del mundo (es decir, marcados socialmente por sus orígenes étnicos/raciales) y las respectivas consecuencias en las relaciones de dominación y explotación (Grosfoguel, 2007). Estos ámbitos son respectivamente la "colonialidad del saber" (Lander, 2000) y la "colonialidad del ser" (Maldonado-Torres, 2007). En términos muy generales, la colonialidad del ser, concepto propuesto y desarrollado por Maldonado-Torres, hace referencia a la implantación de jerarquías del ser, en los que unos seres son prescindibles mientras que otros no lo son. Unas jerarquías que "[...] dividen al mundo entre blancos y sujetos de color en el norte, y entre distintos tipos de mestizos y poblaciones excluidas de proyectos nacionales en el sur" (Maldonado-Torres, 2008).

La colonialidad del saber, por su parte, nos la presenta Edgardo Lander como una clasificación de conocimientos, unos válidos y otros que son invalidados por criterios 
establecidos en el eje de la modernidad, el conocimiento científico moderno se erige como universal y por ende, como el único deseable:

La expresión más potente de la eficacia del pensamiento científico moderno especialmente en sus expresiones tecnocráticas y neoliberales hoy hegemónicas- es lo que puede ser descrito literalmente como la naturalización de las relaciones sociales, la noción de acuerdo a la cual las características de la sociedad llamada moderna son la expresión de las tendencias espontáneas, naturales del desarrollo histórico de la sociedad. La sociedad liberal industrial se constituye -desde esta perspectiva- no sólo en el orden social deseable, sino en el único posible (Lander, 2000: 4).

Partiendo de lo anterior, queda claro entonces que al menos existen formas generales de conocimiento: uno asociado a la modernidad occidental (con diversos matices, por supuesto) y otros que esa cara oculta de la modernidad -la colonialidad del saber- ha subalternizado, en el sentido de invalidar, de poner por debajo del conocimiento moderno, asumiéndolos como inferiores, como no-conocimiento. El conocimiento moderno (el que tuvo el lugar privilegiado de "ser llamado así"), se relaciona al mismo tiempo con un proyecto que según Castro-Gómez consistió en "[...] someter la vida entera al control absoluto del hombre bajo la guía segura del conocimiento" (2000), o con lo que Escobar (2005) llama "logocentrismo occidental moderno", cuando se pregunta acerca de cómo pensar las relaciones de los seres humanos con la naturaleza. Este logocentrismo occidental moderno es:

[...] el proyecto de construir un mundo perfectamente ordenado, racional y predecible... esa tendencia metafísica que identifica la verdad lógica como el fundamento de toda teoría racional del mundo, el cual es constituido por objetos y seres cognoscibles y ordenables... llega al paroxismo, por ejemplo, en la actitud del presidente de Estados Unidos que está dispuesto a destruir la mitad de la tierra para erradicar el mal y establecer el orden perfecto (la USA, por supuesto) (Escobar, 2005: 146).

Este logocentrismo occidental (Escobar, 2005), ese proyecto de la modernidad (Castro-Gómez, 2000), ese paradigma disciplinario (Lanz, 2010) ejerce, desde sus bases epistemológicas, una violencia que ha sido catalogada como invisible (Palermo, 2010), pero que se observa cuando en la práctica se invalidan otros conocimientos producidos por sujetos que no son los protagonistas centrales del relato triunfante y hegemónico de la modernidad, es el conocimiento de los excluidos, aquellos que Fanon llamó "condenados de la tierra" (1963), y estas prácticas se observan tanto en las ciencias naturales como en las ciencias sociales, de manera más velada en las segundas que en las primeras. Caminar hacia la transdisciplinariedad, implica entonces, además de captar la necesidad de trascender disciplinariamente, cuestionar las relaciones de subordinación que ambas caras de la producción de conocimiento moderno establecen con otros conocimientos.

El conocimiento científico occidental moderno considera a los otros conocimientos como "creencias", "religiones" o "cultura", al mismo tiempo que no alcanza a solucionar los problemas de la humanidad en la actualidad, problemas que la modernidad misma de la mano de la ciencia, ha creado. Esto es algo que Sousa Santos (2010) plantea bien cuando dice que tenemos problemas modernos para los cuales no existen soluciones modernas. En un tono similar a Lanz (2010), Sousa Santos nos dice que tomar distancia frente a la modernidad no implica echar a la basura toda esa tradición y el potencial emancipador que representa la modernidad occidental, sino tener la posibilidad de estar dentro y fuera de ella para poder establecer una visión crítica que 
se consolida en lo que denomina sociología de las ausencias, que es una ruta posible para abordar el problema de las relaciones de poder entre sistemas de conocimiento.

Esta sociología de las ausencias consiste en mostrar que "[...] lo que no existe es, de hecho, activamente producido como no existente, o sea, como una alternativa no creíble a lo que existe" (2010). La ciencia occidental moderna ha producido un cuerpo de conocimientos que al mismo tiempo produce como no-existente los demás conocimientos. Conocimientos que ubicados en la matriz de la colonialidad del ser, del saber y del poder, encarnan lo que Blaser y De la Cadena, con Trouillot, ubican en el campo semántico de lo impensable (Blaser y De la Cadena, 2009).

Desde una perspectiva antropológica, las etnografías de prácticas científicas y la observación de contextos en los cuales se pretenden articular (¿articular?) conocimientos científicos con conocimientos indígenas o de otro tipo, también se han ofrecido descripciones y reflexiones que dan lugar a críticas sobre la naturaleza de la modernidad, sobre las tensiones entre estos tipos de conocimientos y por tanto, sobre las maneras en que se entiende o ha definido la realidad o lo real (Blaser, 2009; Blaser y De la Cadena, 2009, Blaser, 2012). Mediante este tipo de etnografías es que también puede hacerse conciencia de nuestras prácticas, máxime cuando la apuesta es, de hecho, la construcción de una agenda que tiene como punto de partida la interdisciplinariedad, la posibilidad de ecología(s) humana(s) -en plural, léase bien- y la existencia de saberes ambientales.

Entonces, parte de lo que ofrece el panorama es una caja de herramientas que combina la etnografía de prácticas científicas, el diálogo de saberes, la ontología política y el compromiso político de descolonizar la universidad como una oportunidad de articular diversos tipos de conocimientos. Existen riesgos, sin embargo. Entre ellos, el de no poder escapar a lo que Eduardo Viveiros de Castro denomina "equivocaciones sin control" y que corresponden a "un tipo de falla comunicativa en la que los interlocutores no están hablando de la misma cosa y no se dan cuenta" (Viveiros de Castro, 2004a, citado en Blaser, 2009) ${ }^{4}$. Otro riesgo no menos importante para tener en cuenta es que no estamos exentos de que las condiciones de diálogo en algunas ocasiones ni siquiera existan, de lo que se desprende el imperativo de construirlas. Con todo, el camino parece esperanzador.

Los planteamientos de Blaser (2009) sobre los conflictos entre el conocimiento occidental moderno y los conocimientos indígenas, hacen referencia a una perspectiva de análisis que toma distancia de lo que desde la ecología política se plantea como un problema entre perspectivas culturales sobre la naturaleza. En lugar de ello, Blaser decide ubicarse por fuera del paradigma del multiculturalismo para explorar estos conflictos siguiendo la senda abierta por Viveiros de Castro en torno al multinaturalismo, es decir, pasando de un asunto de "múltiples culturas" a uno sobre "múltiples naturalezas" (Blaser, 2009) ${ }^{5}$. Así, Blaser plantea que el conflicto no se presenta entre diferentes visiones sobre una única naturaleza o un único mundo -no como un asunto meramente epistemológico-, sino como un conflicto entre naturalezas o mundos, un conflicto ontológico que busca abordar desde lo que llama ontología política (2009).

Adicionalmente, vale la pena resaltar entre los trabajos críticos de la modernidad la teoría del actor-red ${ }^{6}$ (TAR/ANT). Latour es uno de sus principales exponentes (también se encuentran otros investigadores como Mol, 2002; Mol y Law, 2002; Woolgar y Latour, 1979, Martínez-Dueñas, 2012, entre otros). Los trabajos de Latour así como muchos de los planteamientos hechos por trabajos posteriores que siguen su senda, plantean que aquella división característica de la modernidad que pone a la sociedad por un lado y la naturaleza por el otro, en la práctica (en las prácticas científicas) no se 
presenta en la práctica, plagada de factiches. Estos trabajos cuestionan la naturalización de la noción de "lo social" como un tipo de material de realidad de cuyo estudio se encargan las ciencias sociales y siguen la propuesta de, más bien, hablar de asociaciones entre elementos heterogéneos (humanos y no humanos) debido a que lo que existe es una proliferación de relaciones híbridas entre actores que es preciso seguir.

\section{Entramados humano-naturales}

La manera en que comencé este escrito fue mencionando parcamente elementos que constituyen parte en conversaciones que surgen básicamente en términos ecológicos. Podremos estar de acuerdo en que todo aquello que menciono al principio tiene que ver con asuntos ambientales, o mejor, con el asunto de las relaciones entre los seres humanos y sus entornos de vida, además se mencionan elementos que llaman la atención sobre la manera en que pensamos el mundo y lo intervenimos en términos ambientales, económicos y políticos; también se hace cierta alusión a la diversidad cultural y la otredad. Vale hacer una salvedad: tomo la idea de "entramados humanonaturales" de Escobar (2013), un ingeniero químico colombiano que ha sido uno de los grandes exponentes de la antropología del desarrollo, de la teoría social crítica contemporánea y un investigador comprometido con el proceso de comunidades negras (PCN) de Colombia. Con entramados humano-naturales, Escobar hace alusión a la manera en que las relaciones entre humanos y no-humanos toman forma y dan sentido a la vida en un lugar determinado.

De este modo, en el presente aparte echaré mano de la experiencia etnográfica con pescadores del mar de Puebloviejo, Magdalena, un pueblo de pescadores al norte de Colombia. Usaré algunos datos de mi trabajo de campo para ilustrar las relaciones entre estos pescadores y entidades no-humanas que, vistos desde una perspectiva científica -o mejor, desde la ontología moderna y su forma dominante de producir conocimiento- vendrían a ser simplemente aspectos de "la naturaleza", una parte de la realidad que estudian tales como elementos climáticos, astronómicos, creencias o religiones, entre otras cosas.

Al comenzar por los "asuntos climáticos", el vendaval y la brisa son dos corrientes de aire que aquellos hombres de mar distinguen muy bien. Entre otras cosas, "ser hombre de mar ¡sí que tiene implicaciones!, hay que ser fuerte, porque el mar es fuerte". Contando de nuevo, el vendaval es una fuerza que recorre el territorio desde terreno firme hacia afuera, mientras que la brisa recorre el territorio desde afuera hacia la tierra (esto es, lo que desde nuestra lógica asumiríamos como un movimiento desde "mar adentro" hacia la orilla). El vendaval y la brisa caminan el territorio en sentidos contrarios.

Cuando están trabajando "allá afuera" (lo que nosotros asumimos como "mar adentro"), los pescadores tienen que estar atentos a las señales y rastros que dejan el vendaval y la brisa. Cierto tipo de brisa o cierto tipo de vendaval por sus corrientes, los recorridos que hacen o la temperatura que llevan, pueden estar anunciando una inminente tormenta. Los pescadores trabajan dos a bordo de un cayuco ${ }^{7}$, uno es el piloto a quien se le atribuye la sabiduría, el otro es el atarrayero, al que se le atribuye fuerza. La conexión entre los pescadores es una que puede ser consanguínea o por filiación, algunos de ellos sostienen que un ñero, como se le dice a la pareja que dura toda la vida, debe estar dispuesto a ganar o perder la vida con el otro.

La brisa y el vendaval se "pelean" cuando se encuentran. El pescador tiene que seguir sus movimientos y rastros, tiene que "captar sus mensajes" para predecir y escoger el momento exacto en el que sin perder su faena de pesca ni su vida, pueda retirarse. 
Estar en medio de esta pelea puede significar no aparecer más, no regresar a casa. Sin embargo, la brisa es benévola, el vendaval no. Cuando se naufraga la brisa es amiga, lleva al pescador hacia la orilla mientras va rogando que el vendaval no aparezca porque ese se lo lleva "mar adentro".

Más arriba, pero aun haciendo parte del gran territorio ecológico de la Sierra Nevada de Santa Marta, en el Parque Nacional Natural Tairona, los pescadores de una playa llamada Playa del Muerto saben bien lo que la luna quiere decirles y el modo en que influye en el producto de su trabajo (Leal, 2014). La "Iuna clara" (esto es: la luna llena) daña el pescado. Cuando se pesca con luna clara, hay que revisar las capturas a media noche porque si no se hace, se daña y se pierde.

Regresamos al área de la Ciénaga Grande de Santa Marta (CGSM) en el Caribe colombiano, cerca de aquellos "hombres de mar", pero esta vez entrando a zonas llenas de y delimitadas por Avicennia germinans, Rhizophora mangle yLaguncularia racemosa que conforman uno de los siete eco-distritos o ecosistemas de la CGSM, el ecosistema de manglar (Vilardy y González, 2011). Allí, los pueblos de Nueva Venecia y Buenavista han construido sus viviendas con mangle salao, rojo y negro, otros tantos recorren diariamente las geografías que traza el mangle pararelear ${ }^{8}$ y experimentan cada día las posibilidades de cambiar de puesto de trabajo, cambiando alguna de las varas de manglar "para que el otro entienda" Relear implica asumir siempre y en el mismo lugar de trabajo la pelea nocturna que se libra contra jaibas (Callinectes sapidus) y chivos (Ariopsis sp.), para que no se "roben" el camarón del que se alimentan después de vender lo diario en el mercado. ¡Relear es una declaratoria de guerra diaria a peces y crustáceos decápodos!

Jaibas, luna, vientos, gente, agua, chivos, Avicennia germinans, varas de manglar, casas sobre el agua... relaciones entre elementos heterogéneos que conforman entramados humano-naturales. Estos entramados dan cuenta de lo que es estar en lugares distantes, epistemológica, política y ontológicamente de los lugares comunes de la ciencia, de lo que hacemos. Estos entramados humano-naturales cuestionan, desde la encarnación misma de su propia ontología, la naturalización de las posturas que hemos tenido frente a lo ambiental y frente a lo humano, frente a las relaciones entre naturaleza y sociedad y sobre todo, frente a la separación que todavía parece estar guiando algunos de nuestros pasos, si no presionando por mantenerse como sentido común de nuestras prácticas.

\section{Transdisciplina: centrarnos en problemas del mundo nos pone de hecho en un nivel que obliga a trascender la lógica disciplinaria}

Como una forma de reflexión -que no de conclusiones- es necesario tener en cuenta que los trabajos aquí señalados contribuyen a entender la crisis de la modernidad y de la lógica disciplinaria que le es inherente a un sistema de conocimientos que debemos cuestionar y transformar, sin desperdiciar lo que Sousa Santos (2010) refiere como el potencial emancipatorio de la modernidad. A pesar de ello, de tener la perspectiva crítica, de contar con elementos importantes para avanzar en una transformación de las relaciones de poder, corremos el riesgo de repetir o reproducir las jerarquías señaladas tanto por la colonialidad del poder, como por la colonialidad del saber. Los dos marcos teóricos críticos de la modernidad que fueron presentados más arriba, pueden conciliarse siguiendo el rastro del trabajo de Elena Yehia (2008), quien plantea que cada uno de ellos (modernidad/colonialidad/decolonialidad y teoría del actor red) muestra sus lados débiles, complementándose. Por un lado, es importante tener en cuenta que el conocimiento científico es también producto de prácticas y contextos culturales, que hace parte, por decirlo de alguna manera, de una cultura (la cultura occidental y la modernidad); pero por otro, también es preciso contar con claridad 
sobre los procesos históricos mediante los cuales se han invisibilizado e invalidado otros conocimientos.

Los relatos de cuaderno de campo presentados como ejemplo de otras ontologías (pescadores principalmente en el Caribe colombiano) dan cuenta entre otras cosas de conocimientos y relaciones entre humanos y no-humanos diferentes. Estas muchas veces son comentadas como anécdotas entre investigadores y cobran sentido casi que de modo secreto en espacios no-académicos, ignorando las implicaciones políticas de reconocer otras ecologías que no encajan en el modelo moderno. Esto ubica a la antropología en una especie de plataforma exótica y la perpetúa en ese lugar, configurándola ante otras disciplinas como aquella que trata de "asuntos culturales y creencias" de pueblos distintos a los del antropólogo que los describe, poniendo su trabajo casi que en el nivel literario y fantástico y cuyas críticas resultan problemáticas para la racionalidad científica. La antropología requiere ser también otra cosa en el marco de la maestría en Ecología Humana y Saberes Ambientales de la Universidad de Caldas, si quiere cuestionar las lógicas disciplinarias y cientificistas con sus normas de juego, aquellas que deciden qué es y qué no es válido como "realidad". A pesar de esto, los aspectos de la vida de aquellos pueblos, aquellas realidades, terminan siendo contundentes, el vendaval y la brisa siguen determinando patrones de movilidad, orientación y dirección a pescadores en el mar de Puebloviejo, o la luna advierte sobre cómo se debe pescar o cortar el manglar, ordenando las relaciones de producción en la CGSM.

Hago un reclamo para dejar de usar y hablar de estas ontologías como meras anécdotas bonitas y por empezar a establecer una política del conocimiento que reconozca la multiplicidad de mundos -de ecologías humanas en plural, otra vez-, por lo que no se me ocurre una mejor opción que terminar citando a Mario Blaser y Marisol De la Cadena cuando dicen de nosotros los antropólogos:

Como lo señala Paul Nadasdy (2007), en la mayoría de los casos estas historias no son tomadas en serio. En efecto, sólo en las charlas informales, después de las presentaciones en los congresos, nos atrevemos a contar estas historias con un tono circunspecto, dejando abierta la posibilidad de que haya 'algo más-y más allá' de lo que podemos explicar acerca de ellas, desde donde estamos; en público, domesticamos estas historias para hacerlas entrar en nuestros corrales intelectuales, las volvemos 'símbolos,' 'metáforas' es decir 'significado' de expresiones de procesos que ya conocemos, que ya han sido teorizados, es decir, que son pensables: construcciones sociales, animismo, manipulación de símbolos étnicos, y demás. En otras palabras: no podemos pensar las relaciones sociales de las personas a quienes llamamos indígenas sin la ayuda de nuestra antropología moderna o post-moderna. Para eso, después de todo, creamos esa disciplina en el siglo diecinueve y comienzos del veinte: para explicar en nuestros términos, para traducir a nuestro mundo esos otros mundos adonde llegábamos como viajeros avezados, y como liberales bien intencionados deseosos de contribuir a controlar los excesos del proceso civilizador en el que también participábamos. 


\section{Referencias}

- Blaser, M y M, De la Cadena. (2009). “Introducción”. En: World Anthropologies Network / Red de Antropologías del Mundo. Electronic Journal. Num.4, January/Enero. pp. 3 10.

- Blaser, M. (2009). “La ontología política de un programa de caza sustentable”. En: World Anthropologies Network / Red de Antropologías del Mundo. Electronic Journal. Num.4, January/Enero. pp. 81 - 108.

- Castro-Gómez, S. Ciencias sociales, violencia epistémica y el problema de la invención del otro. En: Lander, E. (Comp.). La colonialidad del saber: eurocentrismo y ciencias sociales. Perspectivas latinoamericanas. Consejo Latinoamericano de Ciencias Sociales - CLACSO. Buenos Aires, Argentina. pp. 139 155.

- Escobar, A. (2013). Territorios de diferencia. La ontología política de los “derechos al territorio”. En: Economía Colombiana. Contraloría General de la República. Edición 340. Noviembre-Diciembre. Bogotá, Colombia. pp. 9 - 20.

- Escobar, A. (2005). ¿Cómo pensar la relación entre ser humano y naturaleza?En: Escobar, A. Más allá del Tercer Mundo. Globalización y Diferencia. Instituto Colombiano de Antropología e Historia - ICANH - Universidad del Cauca. pp. 145 -

- Fanon, F. (1963). Los condenados de la tierra. Fondo de Cultura Económica. México D.F.

- Fergusson, A. s.f. Las lógicas disciplinarias y la emergencia de los paradigmas de la complejidad y la transdisciplina. Manuscrito. Hallado en https://miguelangel13.files.wordpress.com/2012/08/las-lc3b3gicas-disciplinarias-y-laemergencia-de-los-paradigmas-de-la-complejidad-y-la-transdisciplina-1.pdf . Revisado el 18 de septiembre de 2015.

- Grosfoguel, Ramón. 2007. Migrantes coloniales caribeños en los centros metropolitanos del sistema-mundo moderno. Los casos de Estados Unidos, Francia, los Países Bajos y el Reino Unido. www.cidob.org Serie Migraciones. Número 13. Junio de 2007. Fundación CIDOB, Barcelona, España.

- Lander, E. (2002). “Ciencias sociales: saberes eurocéntricos y coloniales”. En: Lander, E. (Comp.). La colonialidad del saber: eurocentrismo y ciencias sociales. 
Perspectivas latinoamericanas. Consejo Latinoamericano de Ciencias Sociales CLACSO. Buenos Aires, Argentina. pp. 9 - 38.

- Lanz, R. (2010). Diez preguntas sobre la transdisciplina. En: RET. Revista de Estudios Transdisciplinarios. Vol. 2. Núm. 1. enero - junio, 2010. pp. 11 - 21. Fundación de Estudios Avanzados de Venezuela. Caracas, Venezuela.

- Latour, B. (2007). Nunca fuimos modernos. Ensayo de antropología simétrica. Siglo XXI Editores. Buenos Aires, Argentina.

- Latour, B. (2008). Reensamblar lo social. Una introducción a la teoría del actor red. Manantial. Buenos Aires, Argentina.

- Law, J. y A, Mol. 2002. Complexities. Social studies of knowledge practices.Duke University Press. Durham and London.

- Leal, A. (2015). Ontología política del ecoturismo en el Parque Nacional Natural Tayrona. Entre Playa del Muerto y Playa Cristal. Santa Marta, Colombia. Tesis de pregrado Programa de Antropología de la Universidad del Magdalena.

- Maldonado-Torres, N. (2008). "La descolonización y el giro descolonial” En:Revista Tabula Rasa. Núm. 9. Julio - Diciembre. pp. 61 -72. Universidad Colegio Mayor de Cundinamarca. Bogotá D. C. Colombia.

- Martínez-Dueñas, W. (2012). Cosmopolitismo ambiental y redes multinaturales: un recorrido por mundos no-tan-modernos en Puracé, Colombia. Tesis doctoral. Doctorado en Antropologías Contemporáneas. Universidad del Cauca, Popayán, Cauca, Colombia.

- Mignolo, W. (2008). La opción decolonial. Desprendimiento y apertura. Un manifiesto y un caso. En: Revista Tabula Rasa. Núm. 8. Enero-Junio de 2008. Universidad Colegio Mayor de Cundinamarca. Bogotá D. C. Colombia.

- Mintz, S. (1996). Dulzura y poder. El lugar del azúcar en la historia moderna.Siglo XXI Editores. México.

- Mol, A. (2002). The body multiple. Duke University Press. Durham and London.

- Palermo, Z. 2010. Una violencia invisible: la colonialidad del saber. CUADERNOS FHyCS-UNJu, Nro. 38:79-88. Universidad Nacional de Salta. Argentina.

- Sánchez-Maldonado, J. (2011). Políticas de la pesca ayer y hoy en La Guajira colombiana. Colonialidad del poder, invención del pescador e intervenciones 
biopolíticas y disciplinarias. Tesis de pregrado. Programa de Antropología, Facultad de Humanidades, Universidad del Magdalena. Santa Marta, Colombia.

- Sousa Santos, B. (2010). Descolonizar el saber, reinventar el poder. Ediciones Trilce. Montevideo, Uruguay.

- Yehia, E. (2006). Descolonización del conocimiento y la práctica. Un encuentro dialógico entre el programa de investigación sobre modernidad/colonialidad/decolonialidad latinoamericano y la Teoría del Actor Red. En: Revista Tabula Rasa, Número 6. Pp. 85 - 114. Universidad Colegio Mayor de Cundinamarca. Bogotá D. C. Colombia.

- Quijano, A. (2000). "Colonialidad del poder y clasificación social”. En: Jornal of WolrdSystems Research. Vol. X. Num. 2. Summer -Fall. Special Issue: Festchrift for Immanuel Wallerstein.

- Vilardy, S., y González, J.A. (Eds.). 2011. Repensando la Ciénaga: Nuevas miradas y estrategias para la sostenibilidad en la Ciénaga Grande de Santa Marta. Universidad del Magdalena y Universidad Autónoma de Madrid. Santa Marta, Colombia. 228 p.

- Viveiros de Castro, E. 2004. Perspectival anthropology and the method of controlled equivocation. En: Tipití Journal of the Society for the Anthropology of Lowland South America (2)1: 3-22.

- Wallerstein, I. (1998). "La incorporación de vastas zonas nuevas a la economíamundo" En: Wallerstein, Immanuel. 1998. El moderno sistema mundial. La segunda era de gran expansión de la economía-mundo capitalista 1730-1850. Pp. 179-269. Siglo Veintiuno Editores, Madrid.

- Wallerstein, I. y Quijano, A. (1992). "La americanidad como concepto, o América en el moderno sistema mundial". En: América: 1492 - 1992. Trayectorias históricas y elementos del desarrollo. Revista Internacional de Ciencias Sociales. Vol. XLIV, Número 4. UNESCO. Catalunya. pp. 583 - 592.

- Woolgar, E. y B. Latour. (1979). La vida en el laboratorio. La construcción de los hechos científicos. Alianza Editorial. Madrid, España.

1. Docente investigador Corporación Universitaria Minuto de Dios Vicerrectoría Regional Llanos. Candidato a Magister en Ecología Humana y Saberes Ambientales 
de la Universidad de Caldas. Correo

electrónico: jorge.sanchezm@uniminuto.eduORCID: ORCID: 0000-0001-9200-7821

2. Bruno Latour (2008) define el término actante o actor como entidades que en conexión con otros generan efectos en una red heterogénea de sujetos y objetos en la que cada uno tiene una incidencia en la constitución de la red que los asocia. La investigación tendría que observar y describir la agencia de esos actantes en el proceso de conformación de la red.

3. Para el caso del Caribe colombiano, concretamente La Guajira, vale la pena revisar Sánchez-Maldonado, Jorge. 2011. Políticas de la pesca ayer y hoy en La Guajira colombiana. Colonialidad del poder, invención del pescador e intervenciones biopolíticas y disciplinarias. Tesis de Pregrado. Programa de Antropología, Facultad de Humanidades, Universidad del Magdalena.

4. Blaser (2009) muestra claramente el ejemplo para el caso de un programa de caza sustentable en el Chaco paraguayo, en el que el término "sustentable" implicaba cosas distintas tanto para los conservacionistas como para los indígenas Ishir.

5. Vale recordar que actualmente la Constitución Política de Colombia de 1991, plantea a la nación como multicultural.

6. En español para referirse a la teoría del actor red se usa la sigla TAR, en inglés, se usa la sigla ANT. En este trabajo se usarán ambas indistintamente unidas por una barra inclinada así: TAR/ANT.

7. Con este término se designa a una embarcación hecha de modo artesanal por los mismos pescadores. Distintos de las lanchas que generalmente están hechas en fibra de vidrio, los cayucos comportan todo un conocimiento ancestral transmitido de generación en generación y que por disciplinas como la ingeniería pesquera o la Economía Agrícola de la Universidad del Magdalena, han sido catalogadas como "atrasadas" y "poco eficientes".

8. Con este término se refiere a la actividad de captura de camarones en la Ciénaga Grande de Santa Marta que pone en escena la práctica e implementación de conocimientos ancestrales.

9. Aquí el otro es otro relero, como se le dice a aquellos que se dedican a capturar camarones. Cuando ve que el puesto tiene algo nuevo, es porque alguien está trabajando en ese puesto. Hasta el tiempo en que estuve con los releros, nunca observé que se presentaran problemas por el cambio de puesto.

Para citar este artículo: Sanchez-Maldonado, J. (2017). Entramados humanonaturales como ruta posible hacia la transdisciplinariedad en el campo de la ecología humana y saberes ambientales en la Universidad de Caldas. Revista Luna Azul, 44, 265-276. DOI: 10.17151/luaz.2017.44.16. Recuperado de http://200.21.104.25/lunazul/index.php/component/content/article?id=237

Esta obra está bajo una Licencia de Creative Commons Reconocimiento CC BY

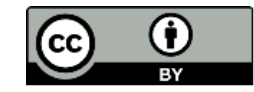

